\title{
Confocal Arthroscope Development For in vivo Knee Joint Diagnosis
}

\author{
D. Smolinski, T.B. Kirk ${ }^{*}$, P. Delaney ${ }^{* * *}$, J.P.Wu*, K. Miller ${ }^{*}$, M. Zheng ${ }^{* *}$.
}

*University of Western Australia, Department of Mechanical and Materials Engineering,

35 Stirling Highway, Crawley, W.A., 6009, Australia

**University of Western Australia, Orthopaedic Surgery (Department of Surgery),

35 Stirling Highway, Crawley, W.A., 6009, Australia

*** OptiScan Pty. Ltd., 15-17 Normanby Rd, Notting Hill, VIC., 3168, Australia

A prototype hand-held confocal arthroscope is under development to assist in the examination of articular cartilage in vivo [1]. Articular cartilage structure is very difficult to study due to its 3D structure interaction and thus little is known about its fundamental function in providing weight bearing strength and lubricating properties in synovial joints. The recent development of a miniaturised confocal laser scanner mechanism by OptiScan Pty. Ltd. (Australia), has made possible the design of a hand held confocal arthroscope. The arthroscope is being developed in collaboration between OptiScan and the Departments of Mechanical and Materials Engineering and Orthopaedic Surgery at UWA with the aim of improving ease of use and functionality for orthopaedic work.

The initial arthroscope probe with outside diameter (OD) of $7 \mathrm{~mm}$ has now been reduced to an OD of $4.4 \mathrm{~mm}$ (Fig. 1, 2), which makes it comparable in size with current video arthroscopes. This is an important step as the instrument is now able to fit into a $5.8 \mathrm{~mm}$ OD trocar used by Orthopaedic surgeons. The imaging performance of the $4.4 \mathrm{~mm}$ OD arthroscope is to be fully tested before a clinical trial prototype is designed although initial results suggest a similar performance to the $7 \mathrm{~mm}$ OD arthroscope which incorporated a 22x objective (aspect ratio of $\sim 1.7$ ), with the field of view being $290 \mu \mathrm{m} \times 480 \mu \mathrm{m}$.

A confocal arthroscope can offer advantages over tissue biopsy for cartilage diagnostic examination since the cartilage in the biopsied region can be slow to heal. The morphological information gained from imaged chondrocytes is hoped to provide real-time information on joint condition $[2,3]$. The arthroscope's image acquisition specifications so far have proven adequate to perform structural studies of the cartilage in human post mortem specimens (Fig. 3, 4) as well as in vivo rabbit joints.

This paper focuses on the technical challenges faced with this new imaging technology and discusses a complete knee joint confocal diagnosis system. Other technical requirements for clinical application in an orthopaedic surgery will also be discussed.

[1] Smolinski D, et al, The $3^{\text {rd }}$ Asian-Pacific Symposium on Confocal Microscopy and Related Technologies, (2001), 70.

[2] Wu J P, et al, Unpublished, (2002).

[3] Wu J P, et al, European Society Biomechanics Conference, (2000), 209. 


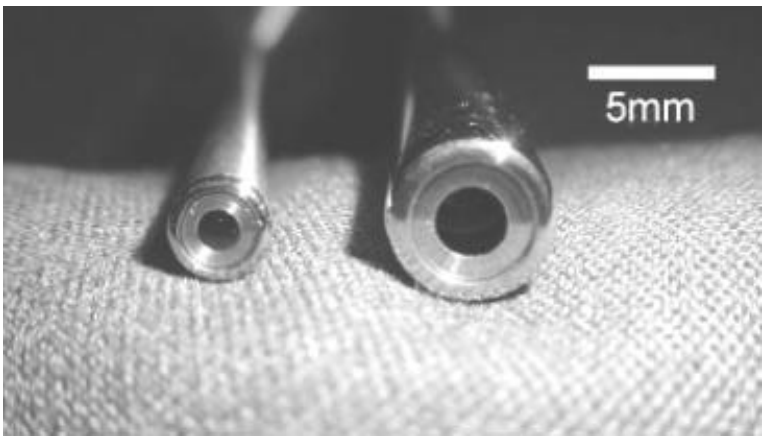

Figure 1: Comparison of the $4.4 \mathrm{~mm}$ OD confocal arthroscope with the $7 \mathrm{~mm}$ OD confocal arthroscope

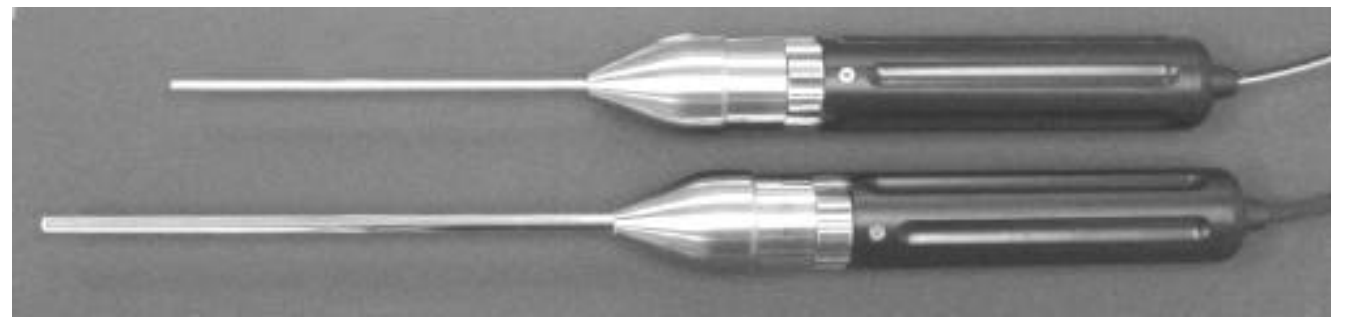

Figure 2: The two prototype confocal arthroscopes side by side

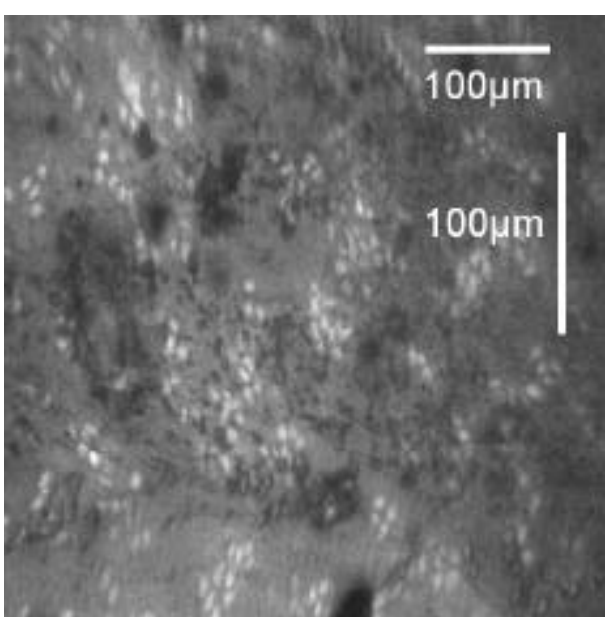

Figure 3:

Articular cartilage from the weight-bearing region (using $7 \mathrm{~mm}$ OD)

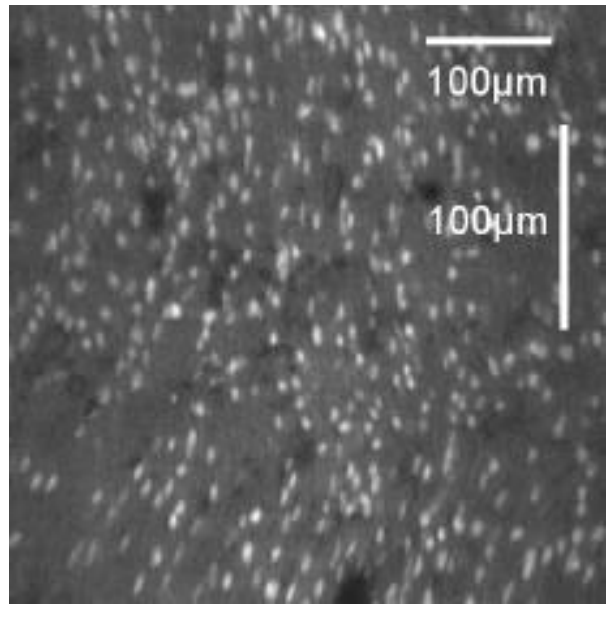

Figure 4:

Femoral head meniscus (using $7 \mathrm{~mm}$ OD) 